УДК 004.932.2+ 665.6/.7

\title{
АДАПТИВНАЯ ИНФОРМАЦИОННО-ИЗМЕРИТЕЛЬНАЯ СИСТЕМА ДЛЯ МОНИТОРИНГА ПРОТЕКАНИЯ ФИЗИКО-ХИМИЧЕСКОГО ПРОЦЕССА
}

\author{
Цавнин Алексей Владимирович', \\ avc14@tpu.ru
}

\section{Филипас Александр Александрович 1 filipas@tpu.ru}

\author{
Беляев Александр Сергеевич ${ }^{1}$, \\ asb22@tpu.ru
}

\author{
Рожнев Никита Викторович1, \\ rozhnevn@gmail.com \\ 1 Национальный исследовательский Томский политехнический университет,
Россия, 634050, г. Томск, пр. Ленина, 30.
}

\begin{abstract}
Актуальность исследования обусловлена необходимостью повышения экономической рентабельности в нефртегазовом секторе за счет автоматизированного управления технологическими процессами разделения нефртесодержащей жидкости на основе математических имитационных моделей, полученных на основе натурных экспериментов, в частности, снижение энергетических затрат на обеспечение данных технологических процессов без потери качества товарной нефрти, регламентируемого государственными стандартами.

Цель: создание адаптивной автоматизированной информационно-измерительной системы для экспериментальной оценки динамики сепарации нефтесодержащей жидкости при различной степени устойчивости слоя эмульсии и межфракционной дифффузии для формирования данных для построения имитационной математической модели.

объект: технологический процесс сепарации нефтесодержащей жидкости на примере лабораторного резервуара с гравитационным типом сепарации.

Методы: натурный эксперимент, техническое зрение, свёрточные нейронные сети, машинное обучение, сегментация цифррового изображения, расчет объемных соотношений фракций, переходные процессы, автоматизация физико-химического эксперимента.

Результаты. Разработана адаптивная информационно-измерительная система на базе технического зрения и свёрточных нейронных сетей, позволяющая производить оценку объемного соотношения разделенных фрракций нефртесодержащей жидкости при различных конфигурациях слоя эмульсии и разной степени его стабильности и четкости границы. Основой функционирования представленной информационно-измерительной системы является программное обеспечение, предусматривающее различные качественные и количественные параметры проведения эксперимента. Разработан и представлен алгоритм адаптации периода дискретизации фотофиксации на основе продолжительности фризико-химической реакции, определяемой постоянной времени процесса. Для представленной адаптивной информационно-измерительной системы проведена апробация в рамках лабораторного эксперимента с получением объемных соотношений фрракций в режиме реального времени и их визуализацией.
\end{abstract}

\section{Ключевые слова:}

Cenаратор, нефртесодержащая жидкость, эмульсия, компьютерное зрение, свёрточные нейронные сети, адаптивное измерение.

\section{Введение}

На сегодняшний день в нефтедобывающем секторе одним из ключевых элементов первичной обработки нефтесодержащей жидкости является сепаратор устройство, в котором нефть отделяется от попутного газа, воды и иных примесей. Одним из наиболее распространенных видов сепараторов является сепаратор гравитационного типа, в котором процесс разделения обеспечивается за счет различной плотности жидкостей [1-3]. В свою очередь, среди сепараторов гравитационного принципа действия выделяют двухфазные и трехфазные, в зависимости от количества фракций, которые могут быть зафиксированы и измерены во время процесса сепарации скважной жидкости [4-7]. В трехфазном сепараторе скважная жидкость разделяется на три ключевые фракции - вода, нефть и газ.
Для обеспечения автоматизированного и оптимального в смысле быстродействия, энергозатрат и износа технологического оборудования регулирования необходимо наличие формализованного математического описания процесса разделения скважной жидкости. На сегодняшний день существует целый ряд работ [8-13], посвященных оценке и формализации процесса разделения скважной жидкости. Наиболее простым способом получения математической модели динамики сепарации является проведение эксперимента с получением имитационной модели, т. е. аппроксимация некоторой функцией набора измеренных значений толщины каждого из слоев. Для определения объемного соотношения полученных фракций в промышленности применяются измерители уровня с возможностью детектирования границы раздела сред, однако совокупность фаз уже разделен- 
ной скважной жидкости характеризуется неравномерными границами, т. е. слой может иметь клиновидную форму или более сложную. В условиях, максимально приближенным к реальным технологическим процессам, переход между фракциями может не иметь четкой границы

Для более точной фиксации динамики процессов разделения с помощью измерителей с возможностью детектирования границ раздела фаз на одном объекте требуется несколько датчиков, что существенно увеличивает стоимость эксперимента. Кроме того, есть более сложные системы измерения, позволяющие учитывать нестабильность слоя эмульсии [14]

Качественно другим подходом к решению задачи оценки динамики процесса разделения может являться применение системы технического зрения в рамках предварительного лабораторного эксперимента. Предлагается решение задачи сегментации цифрового изображения на основе данных о значении интенсивности пикселей, получаемых из видеопотока. Однако стоит отметить, что осуществление оценки объемного соотношения фракций в условиях добывающей площадки, в силу того, что сепаратор представляет собой непрозрачный металлический резервуар без непосредственного доступа к прямому оптическому наблюдению за состоянием фаз с применением технического зрения, является отдельной инженерной задачей. Решением для проведения эксперимента с оптическим наблюдением за процессом может являться построение испытательного стенда, который представляет собой модель физического подобия реального промышленного сепаратора, но в конструктивном исполнении, позволяющем вести прямое наблюдение. В данном случае предлагается построение накопителя из прозрачного органического стекла или пластика.

\section{Описание эксперимента}

Для оценки динамики процесса сепарации скважной жидкости резервуар, пригодный для непрерывной оптической оценки, наполняется смесью воды и заранее заготовленной сырой нефтью или заменителем. В данной работе в качестве заменителя выступает масло. Далее, непосредственно перед резервуаром, располагается цифровая камера, осуществляющая захват видеопотока. Видеопоток поступает на электронно-вычислительную машину для оценки объемного соотношения каждой из фракций и логирования данных с их дальнейшей визуализацией и математической обработкой. Принципиальная схема эксперимента приведена на рис. 1.

В качестве устройства обработки видеоинформации выбран микрокомпьютер NVIDIAJetsonNano, обеспечивающий должный уровень производительности в задачах параллельных вычислений, распознавания образов и сегментации.

Представленный алгоритм функционирования системы позволяет осуществлять автоматизированное наблюдение за протеканием эксперимента с фиксацией ключевых показателей, в данном случае - объемные соотношения фракций нефтесодержащей жидко- сти с их дальнейшим логированием. Кроме того, предусмотрено проведение серии экспериментов в автоматическом режиме, который по истечении выбранного при запуске времени опыта или при получении иной обратной связи, например, при достижении некоторых физико-химических или техникоэкономических показателей, осуществляет перезапуск эксперимента и его проведение выбранное число раз.

Разработанное программное обеспечение (ПО) работает согласно алгоритмам, представленным на рис. 2.

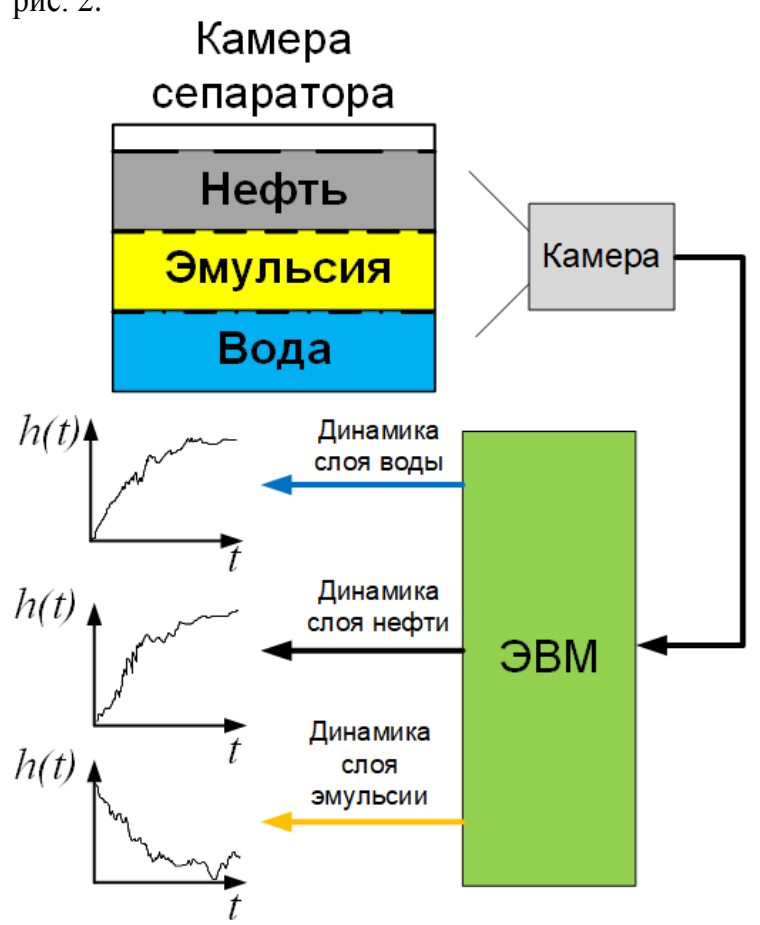

Рис. 1. Приниипиальная схема эксперимента

Fig. 1. Experiment flow chart

При запуске программы пользователь выбирает режим работы: одиночный эксперимент или серия экспериментов. В случае выбора пользователем одиночного эксперимента далее задается длительность эксперимента и желаемое значение точности. Исходя из введенных значений входных параметров, осуществляется автоматический расчет частоты фотофиксации. После этого в течение всего времени проведения эксперимента циклически осуществляется захват видеопотока с его дальнейшим кадрированием, сегментированием, выделением границ и расчетом площадей сегментов и, соответственно, объемных соотношений фракций. В случае выбора серии экспериментов пользователь указывает длину серии, а также выбирает будет ли осуществляться автоматическая адаптация по частоте фотофиксации. В режиме без адаптации осуществляется проведение выбранного количества одиночных экспериментов с указанной длительностью и желаемой точностью. В случае если адаптация по периоду фотофиксации активирована, на первом эксперименте в серии осуществляется расчет постоянной времени процесса и дальнейший перерасчёт периода фотофиксации для более рационального использования вычислительных мощностей ЭВМ. 



Рис. 2. Блок-схемы алгоритмов (А) основной программы и (Б) процедуры оптического мониторинга

Fig. 2. Block diagram of $(A)$ main program and $(B)$ optical monitoring procedure

\section{Процедура оценки объемного соотношения} с помощью компьютерного зрения

Для решения задачи сегментации имеется множество работ, однако наибольшее применение получили сверточные нейронные сети (CHC), которые показывают наибольшую точность в задачах обработки изображения $[15,16]$. Основной особенностью СНС является элемент свертки, который уменьшает исходное изображение до определенных размеров.

Для корректного обучения СНС требуется обширная размеченная выборка данных, что в ряде случае является крайне трудоемкой в плане времени задачей. Одним из подходов к решению данной задачи является трансферное обучение [17-19]. Метод трансферного обучения подразумевает использование уже обученной $\mathrm{CHC}$, у которой меняются и переобучаются только выходные слои, соответственно, для используемой CHC была выбрана U-net архитектура. Выбранная архитектура позволяет осуществить сегментацию области изображения по классу при помощи создания маски каждого из искомых сегментов.

Для обучения СНС была сделана выборка из 100 фотографий, отличающихся по освещению, положению и углу поворота камеры, размытию и с разными конфигурациями эмульсии. В каждом блоке данных для обучения используется по 10 изображений, по которым происходила настройка параметров СНС, значение коэффициента скорости обучения (КСО) в начале было установлено 0,005 и далее значение КСО уменьшалось в 10 раз через каждые 10 блоков данных. Однако через каждые 10 эпох коэффициент уменьшался в 10 раз. Максимальное количество эпох обучения равно 60.

Таким образом, алгоритмы компьютерного зрения настроены на сегментацию ключевых фракций, каждая из которых является эмульсией с различной концентрацией масла.

На рис. 3 представлено исходное изображение резервуара и соответствующие тепловые карты для каждого из слоев, которые получились в результате сегментации. Фракция № 1 является фракцией с самой высокой концентрацией масла и соответствует нефтяному слою после коалисценции, вызванной гравитационным отстаиванием, фракция № 2 представляет собой переходную эмульсионную зону или «промслой». Фракция № 3 является эмульсией с повышенной степенью обводненности. Тепловые карты показывают принадлежность каждого из пикселей к той или иной из фракций. Затем на основе данных тепловой карты формируется так называемая маска - специальное изображение, которое однозначно выделяет ключевые сегменты.

Полученная нейронная сеть имеет точность сегментации не менее $95 \%$, а в среднем - $97 \%$ [20, 21].

Для оценки объема каждой из фракций и, соответственно, динамики сепарации осуществляется расчет площади детектированного сегмента и далее, зная габариты и форму экспериментального резервуара, осуществляется вычисление объема. Данный подход позволяет получить значения объемов в условиях нелинейной формы границы раздела фракций и пониженной стабильности слоя эмульсии, вызванной межфракционной диффузией. 


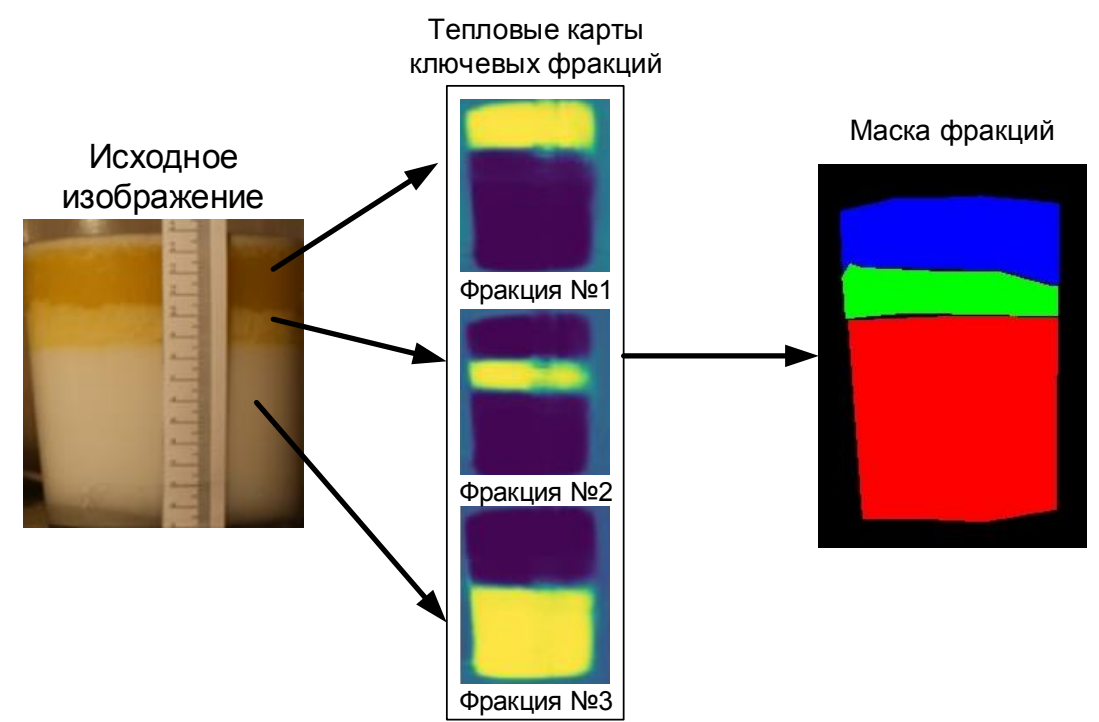

Рис. 3. Выделение фракиий скважной жидкости на основе работы сверточной нейронной сети

Fig. 3. Petroleum-containing fluid fractions recognition using convolutional neural network



Pис. 4. Графики изменения объемных соотношений каждой из фракиий

Fig. 4. Graphics of volume ration of each fraction versus time

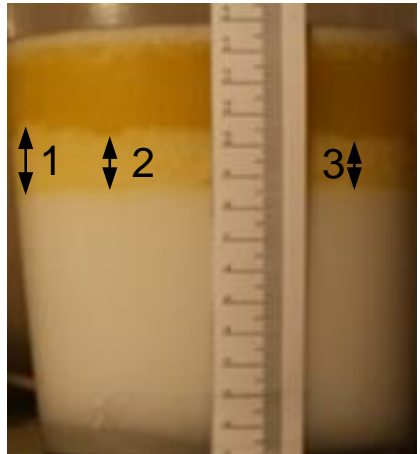

Рис. 5. Сопоставление значений высоты промслоя в зависимости от места измерения

Fig. 5. Comparison of intermediate emulsion layer heights in different locations
Таблица. Высота промслоя при различном расположении измерителя

Table. Intermediate emulsion layer height in different sensor locations

\begin{tabular}{|c|c|}
\hline $\begin{array}{c}\text { Номер метки измерения } \\
\text { Measurement label number }\end{array}$ & $\begin{array}{c}\text { Значение высоты слоя, мм } \\
\text { Layer height, mm }\end{array}$ \\
\hline 1 & 24 \\
\hline 2 & 17 \\
\hline 3 & 17 \\
\hline
\end{tabular}

Важно отметить, что представленный подход на основе компьютерного зрения позволяет нивелировать клиновидность слоя и, соответственно, снизить погрешность измерения. Рассмотрим процесс прямого измерения в данном эксперименте и на основе шкалы оценим высоту промслоя. 
Как видно из рис. 5 и таблицы, в силу клиновидности промслоя, в зависимости от расположения измерителя, имеем различные показания с существенным разбросом. Кроме того, в динамическом режиме работы, т. е. при условии нестатической жидкости, подверженной коалисценции, а при постоянной циркуляции, характерным побочным эффектом будет являться волнообразование, которое также будет увеличивать девиации сигнала измерения. При использовании компьютерного зрения оценка происходит по площади сечения слоя, независимо от его геометрической формы в реальном масштабе времени.

\section{Процедура адаптации периода фотофиксации процессов}

При получении сырых данных о площади каждой из фракций скважной жидкости и оценке ее динамики система адаптирует частоту фиксации изображения, что позволяет уменьшить вычислительную нагрузку на ЭВМ. В силу того, что время разделения скважной жидкости на фракции, с учетом соотношения фракций, температуры и химических свойств компонентов может составлять от нескольких минут до нескольких часов, минимальный период дискретизации $T_{\min }$, т. е. фиксирование состояния эмульсии оптическим устройством, был выбран равным 10 секундам. Как видно на рис. 4, на участке 1 динамика расслоения фракций существенна и для повышения точности эксперимента частота снятия данных будет максимальной. На стадии 2 на рис. 4 дифференциация также существенная, однако наблюдается более низкая динамика формирования слоев каждой из фракций, что допускает снижение частоты захвата кадра. Наконец, на участке 3 динамика снизилась почти до нулевого значения и для более рационального использования ЭВМ в плане вычислительной нагрузки период фотофиксации будет снижен. В силу того, что процессы формирования

\section{СПИСОКЛИТЕРАТУРЫ}

1. Кравцов А.В., Ушева Н.В., Бешагина Е.В. Технологические основы и моделирование процессовпромысловой подготовки нефти и газа: учебное пособие. - Томск: Изд-во Томского политехнического университета, 2012. - 128 с

2. Арнольд К.Е., Стюарт М.И. Справочник по оборудованию для комплексной подготовки нефти. - М.: Премиум Инжиниринг, 2011. $-776 \mathrm{c}$.

3. Щодро А.И. Автоматизация технологического процесса сепарации нефтесодержащей смеси // Актуальные проблемы науки и образования в современном мире: тр. III Международной научно-практической конференции. - Стерлитамак: СФ БашГУ, 2017. - С. 407-410.

4. Автономный комплекс управления трёхфазным нефтегазосепаратором // ЗАО «Альбатрос». URL: http://www.albatros.ru/ catalog/products/mpc-oil-products/control-system-3-phase.php (дата обращения 27.03.2020).

5. Верёвкин А.П., Кирюшин О.В., Уразметов Ш.Ф. Управление процессом подготовки нефти по агрегативной устойчивости водонефтяной эмульсии // Автоматизация, телемеханизация и связь в нефтяной промышленности. - 2012. - № 4. - С. 33-35.

6. Гаврилов В.П. Концепция продления «нефтяной эры» России // Геология нефти и газа. -2005 . - № 1. - С. 53-59.

7. Varanasi S.K., Tirumalaraju S.V., Jampana P. Comparative study of parsimonious NARX models for three phase separator // 2019 Fifth Indian Control Conference (ICC). - New Delhi, 2019. P. 430-435. фракций нефтесодержащей жидкости могут быть описаны как экспоненциальные, формализованная оценка динамики может осуществляться на основе значения постоянного времени процесса $T$, т. е. времени, за которое амплитуда достигнет значения 63,2 \% или снизится от $100 \%$ до значения 36,8\% [22]. В качестве опорного значения для подстройки периода дискретизации будем использовать постоянную времени самого медленного из процессов для отдельно взятого эксперимента. Кроме того, известно, что время достижения 98,2 \% своей амплитуды для экспоненциального процесса составляет порядка 5T. Отсюда период дискретизации $T_{\Phi}$, т. е. в данном случае величина временных интервалов, через которые осуществляется фотофиксация процесса, будет определяться следующим образом

$$
T_{\Phi}=\left\{\begin{array}{l}
T_{\min }, 0 \leq t<T ; \\
2 T_{\min }, T \leq t<3 T \\
5 T_{\min }, t \geq 3 T
\end{array}\right.
$$

\section{Заключение}

Разработанная система технического зрения позволяет в режиме реального времени автоматизировать научный эксперимент по разделению нефтесодержащей жидкости в лабораторных условиях. Представленная информационно-измерительная система позволяет сегментировать фракции с различной степенью стабильности слоя эмульсии и, соответственно, предоставлять уточненные данные для дальнейшего построения имитационной или других типов математических моделей. Кроме того, разработанное программное обеспечение может являться универсальным инструментом для построения статических характеристик, мониторинга и оценки динамики различных наблюдаемых физических, химических и биологических процессов и реакций.

8. Tuning a three-phase separator level controller via particle swarm optimization algorithm / L. Sathasivam, I. Elamvazuthi, M.K.A.A. Khan, S. Parasuraman // 2018 International Conference on Recent Trends in Electrical, Control and Communication (RTECC). - Malaysia, 2018. - P. 265-268.

9. Janakiraman S., Devanathan R. An analysis of quadratic linearization of three phase horizontal gravity separator // 5th International Conference on Control, Decision and Information Technologies (CoDIT). - Thessaloniki, 2018. - P. 1006-1011.

10. Модульный принцип построения математических моделей аппаратов и технологических схем промысловой подготовки нефти / С.Ф. Ким, Н.В. Ушева, М.А. Самборская, О.Е. Мойзес, Е.А. Кузьменко // Нефтепереработка и нефтехимия. - 2013. № 10. - C. 41-44

11. Modelling of dewatering and desalting processes for largecapacity oil treatment technology / S.F. Kim, N.V. Usheva, O.E. Moyzes, E.A. Kuzmenko, M.A. Samborskaya, E.A. Novoseltseva // Procedia Chemistry. - 2014. - V. 10. P. 448-453.

12. Soo H., Radke C.J. A filtration model for the flow of dilute, stable emulsions in porous media // Chemical Engineering Science. 1986. - V. 41. - P. 263-272.

13. Sayakhov F.L., Imashev N.Sh., Fatykhov M.A. Interaction of highly stable water-oil emulsion with radio frequency electromagnetic field // Heavy Crude and Tar Sands Hydrocarbons for the $21^{\text {st }}$ Century: $5^{\text {th }}$ UNITAR International Conference on Heavy Crude and Tar Sands. - Caracas: Petroleos de Venezuela, S.A., 1991. - P. 507-513. 
14. Жданов О.П., Шаталов В.И. Система измерения уровня и межфазных границ многокомпонентных продуктов УМФЗ00 в решении актуальных технологических задач подготовки нефти // Сфера нефтегаз. Приборостроение. - 2011. - № 1. C. $34-40$.

15. Wang R.J., Li X., Ling C.X. Pelee: a real-time object detection system on mobile devices // 32nd Conference on Neural Information Processing Systems (NeurIPS 2018). - Montréal, Canada, 2018. - P. 1-10.

16. Mobile nets: efficient convolutional neural networks for mobile vision applications / A.G. Howard, M. Zhu, B. Chen, D. Kalenichenko, W Wang., T. Weyand, M. Andreetto, H. Adam // arXiv:1704.04861v1 [cs.CV]. URL: https://arxiv.org/abs/1704.04861 (дата обращения 18.01.2020)

17. Simonyan K., Zisserman A. Very deep convolutional networks for large-scale image recognition // 3rd International Conference on Learning Representations, ICLR 2015. - San Diego, 2015. - P. 1-14

18. Szegedy C. et al. Going deeper with convolutions // Proceedings of the IEEE Computer Society Conference on Computer Vision and Pattern Recognition 2015. - Boston, MA, 2015. - P. 1-9.
19. Ronneberger O., Fischer P., Brox T. U-Net: convolutional networks for biomedical image segmentation // arXiv:1505.04597v1 [cs.CV] URL: https://arxiv.org/pdf/ 1505.04597.pdf (дата обращения 03.03.2020).

20. Shajkofci A., Liebling M. Spatially-variant CNN-based point spread function estimation for blind deconvolution and depth estimation in optical microscopy // IEEE Transactions on Image Processing. - 2020. - V. 29. - P. 5848-5861.

21. Low-precision CNN model quantization based on optimal scaling factor estimation / Y. Zhou, L. Chen, R. Xie, L. Song, W. Zhang // 2019 IEEE International Symposium on Broadband Multimedia Systems and Broadcasting (BMSB). - Jeju, South Korea, 2019. P. 1-5.

22. Федоров Ю.Н. Справочник инженера по АСУТП: проектирование и разработка. - М.: Инфра-Инженерия, 2008. - 928 с.

Поступила 04.09.2020 2.

\section{Информация об авторах}

Цавнин А.В., аспирант отделения автоматизации и робототехники Инженерной школы информационных технологий и робототехники Национального исследовательского Томского политехнического университета.

Филипас A.A., кандидат технических наук, доцент отделения автоматизации и робототехники Инженерной школы информационных технологий и робототехники Национального исследовательского Томского политехнического университета.

Беляев $\boldsymbol{A . C . , ~ а с п и р а н т ~ о т д е л е н и я ~ а в т о м а т и з а ц и и ~ и ~ р о б о т о т е х н и к и ~ И н ж е н е р н о и ̆ ~ ш к о л ы ~ и н ф о р м а ц и о н н ы х ~ т е х - ~}$ нологий и робототехники Национального исследовательского Томского политехнического университета.

Рожнев H.B., студент отделения автоматизации и робототехники Инженерной школы информационных технологий и робототехники Национального исследовательского Томского политехнического университета. 
UDC 004.932.2+665.6/.7

\title{
ADAPTIVE INFORMATION AND MEASUREMENT SYSTEM FOR MONITORING PHYSICAL AND CHEMICAL PROCESS BEHAVIOR
}

\author{
Alexey V. Tsavnin ${ }^{1}$, \\ avc14@tpu.ru
}

\author{
Alexander A. Filipas ${ }^{1}$, \\ filipas@tpu.ru
}

Alexander S. Belyaev1,
asb22@tpu.ru

\section{Nikita V. Rozhnev',} rozhnevn@gmail.com

\author{
1 National Research Tomsk Polytechnic University, \\ 30, Lenin avenue, Tomsk, 634050, Russia.
}

The relevance of the research is caused by the need of profitability improving in oil and gas sector with automated control of petroleumcontaining fluid separation, particularly, energy cost reduction without tank oil quality loss. The automated control can be built on the basis of mathematical models that can be obtained with natural experiment.

The main aim of the research is to develop adaptive automated information and measurement system for experimental petroleumcontaining fluid separation dynamics estimation with different water-in-oil emulsion layer stability and interbed diffusion for data collection for mathematical model development.

Object: technological process of petroleum-containing fluid separation in the context of lab bench on the gravity type separation basis Methods: natural experiment, computer vision, convolutional neural networks, machine learning, digital image segmentation problem, volume ration calculation, transient processes, physics and chemistry experiment automation.

Results. The authors have developed an adaptive information and measurement system on computer vision and convolutional neural networks basis, which allows estimating petroleum-containing fluid separation dynamics with different water-in-oil emulsion layer stability and sharpness of layers borders. The basis of functioning of adaptive information measurement system is software, that allows performing experiment considering different qualitative and quantitative conditions. The paper considers the algorithm for discretization period with respect to reaction length. The system was tested on lab bench and layers volume ratio was calculated in real-time.

\section{Key words:}

Separator, petroleum-containing fluid, emulsion, computer vision, convolutional neural networks, adaptive measurement.

\section{REFERENCES}

1. Kravtsova A.V., Usheva N.V., Beshagina E.V. Tekhnologicheskie osnovy i modelirovanie protsesov promyslovoy podgotovki nefti $i$ gaza [Technological basics and modeling of trade oil and gas processing].Tomsk, TPU Publ. house, 2012. 128 p.

2. Arnold K.E., Stuart M.I. Spravochnik po oborudovaniyu dlya kompleksnoy podgotovki nefti [Equipment handbook for complex oil processing]. Moscow, Premium Engineering Publ., 2011. 776 p.

3. Schodro A.I. Avtomatizatsia tekhnologicheskogo protsessa separatsii neftesoderzhashchey zhidkosti [Petroleum-containing fluid separation process automation]. Aktualnye problemy nauki i obrazovaniya v sovremennom mire. Trudy III Mezhdunarodnoy nauchno-prakticheskoy konferentsii [Relevant problems of science and education in modern world: proc. of III International science and practice conference]. Sterlitamak, SF BashSU Publ., 2017. pp. 407-410.

4. Avtonomny kompleks upravleniya trekhfaznym separatorom [Autonomous complex of controlling three-phase separator]. Available at: http://www.albatros.ru/catalog/products/mpc-oilproducts/ control-system-3-phase.php (accessed 27 March 2020).

5. Verevkin A.P., Kiryushkin O.V., Urazmetov S.F. Upravlenie protsessom podgotovki hefti po agregativnoy ustoichivoy vodoneftyanoy emulsii [Control for oil processing with aggreagative stable water-oil emulsion]. Avtomatizatsiya, telemehanizatsiya $i$ svyaz $v$ neftyanoi promyslennosti, 2012, no. 4 pp. 33-35.

6. Gavrilov V.P. Kontseptsiya prodleniya «neftyanoy ery» Rossii [Prolongation of Russia's «petroleum era»]. Geology of oil and gas, 2005, no. 1, pp. 53-59.
7. Varanasi S.K., Tirumalaraju S.V., Jampana P. Comparative study of parsimonious NARX models for three phase separator. 2019 Fifth Indian Control Conference (ICC). New Delhi, 2019. pp. 430-435.

8. Sathasivam L., Elamvazuthi I., Khan M.K.A.A., Parasuraman S. Tuning a three-phase separator level controller via particle swarm optimization algorithm. 2018 International Conference on Recent Trends in Electrical, Control and Communication (RTECC). Malaysia, 2018. pp. 265-268.

9. Janakiraman S., Devanathan R. An analysis of quadratic linearization of three phase horizontal gravity separator. $5^{\text {th }}$ International Conference on Control, Decision and Information Technologies (CoDIT). Thessaloniki, 2018. pp. 1006-1011.

10. Kim S.F., Usheva N.V., Samborskaya M.A., Moyzes O.E., Kuzmenko E.A. Modular principle of construction of mathematical models of apparatuses and flow charts of oil trade preparation. Oil Processing and Petrochemistry, 2013, vol. 10, pp. 41-44. In Rus.

11. Kim S.F., Usheva N.V., Moyzes O.E., Kuzmenko E.A., Samborskaya M.A., Novoseltseva E.A. Modelling of dewatering and desalting processes for large-capacity oil treatment technology. Procedia Chemistry, 2014, vol. 10, pp. 448-453.

12. Soo H., Radke C.J.A filtration model for the flow of dilute, stable emulsions in porous media. Chemical Engineering Science, 1986, vol. 41, pp. 263-272.

13. Sayakhov F.L., Imashev N.Sh., Fatykhov M.A. Interaction of highly stable water-oil emulsion with radio frequency electromagnetic field. Heavy Crude and Tar Sands -Hydrocarbons For the 21-st Century. 5-th UNITAR International Conference on Heavy Crude and Tar Sands. Caracas, Petroleos de Venezuela, S.A., 1991. pp. 507-513.

14. Zhdanov O.P., Shatalov V.I. Sistema izmereniya urovnya i mezhfaznykh granits mnogokomponentnykh produktov [Level and 
interphase borders measurement system UMF300 for multicomponent products]. Sfera neftegaz. Priborostroenie, 2011, no. 1 , pp. 34-40.

15. Wang R.J., Li X., Ling C.X. Pelee: a real-time object detection system on mobile devices. $32^{\text {nd }}$ Conference on Neural Information Processing Systems (NeurIPS 2018). Montréal, Canada, 2018. pp. $1-10$.

16. Howard A.G., Zhu M, Chen B., Kalenichenko D., Wang W, Weyand T., Andreetto M., Adam H. MobileNets: Efficient Convolutional Neural Networks for Mobile Vision Applications. ArXiv:1704.04861v1 [cs.CV]. Available at: https://arxiv.org/abs/ 1704.04861 (accessed 18 January 2020).

17. Simonyan K., Zisserman A. Very deep convolutional networks for large-scale image recognition. $3^{\text {rd }}$ International Conference on Learning Representations, ICLR 2015. San Diego, 2015. pp. 1-14.

18. Szegedy C. Going deeper with convolutions. Proc. of the IEEE Computer Society Conference on Computer Vision and Pattern Recognition 2015. Boston, MA, 2015. pp. 1-9.
19. Ronneberger O., Fischer P., Brox T. U-Net: Convolutional Networks for Biomedical Image Segmentation. ArXiv:1505.04597v1 [cs.CV] Available at: https://arxiv.org/pdf/1505.04597.pdf (accessed 3 March 2020).

20. Shajkofci A., Liebling M. Spatially-variant CNN-based point spread function estimation for blind deconvolution and depth estimation in optical microscopy. IEEE Transactions on Image Processing, 2020, vol. 29, pp. 5848-5861.

21. Zhou Y., Chen L., Xie R., Song L., Zhang W. Low-precision CNN Model Quantization based on Optimal Scaling Factor Estimation. 2019 IEEE International Symposium on Broadband Multimedia Systems and Broadcasting (BMSB). Jeju, South Korea, 2019. pp. $1-5$.

22. Fedorov Y.N. Spravochnik inzhenera po ASU TP. Razrabotka $i$ proektirovanie [Control engineer handbook. Design and development]. Moscow, Infra-Inzheneriya Publ., 2008. 928 p.

Received: 4 September 2020.

\section{Information about the authors}

Alexey V. Tsavnin, postgraduate student, assistant, National Research Tomsk Polytechnic University.

Alexander A. Filipas, Cand. Sc., associate professor, National Research Tomsk Polytechnic University.

Alexander S. Belyaev, postgraduate student, assistant, National Research Tomsk Polytechnic University. Nikita V. Rozhnev, student, National Research Tomsk Polytechnic University. 